九州大学学術情報リポジトリ

Kyushu University Institutional Repository

\title{
Using Maximal Independent Sets to Solve Problems in Parallel
}

Shoudai, Takayoshi

Department of Control Engineering and Science Kyushu Institute of Technology

Miyano, Satoru

Research Institute of Fundamental Information Science Kyushu University

http://hdl. handle. net/2324/3143

出版情報: RIFIS Technical Report. 36, 1991-02-18. Research Institute of Fundamental Information Science, Kyushu University バージョン :

権利関係 : 


\section{RIFIS Technical Report}

\section{Using Maximal Independent Sets to Solve Problems in Parallel}

Takayosi Shoudai

Satoru Miyano

February 18, 1991

Research Institute of Fundamental Information Science

Kyushu University 33

Fukuoka 812, Japan 


\title{
Using Maximal Independent Sets to Solve Problems in Parallel
}

\author{
Takayoshi Shoudai \\ Department of Control Engineering and Science \\ Kyushu Institute of Technology, Iizuka 820, Japan \\ E-mail:shoudai@ces.kyutech.ac.jp \\ Satoru Miyano \\ Research Institute of Fundamental Information Science \\ Kyushu University 33, Fukuoka 812, Japan \\ E-mail:miyano@rifis.sci.kyushu-u.ac.jp
}

\begin{abstract}
By using an $O\left((\log n)^{2}\right)$ time EREW PRAM algorithm for a maximal independent set problem (MIS), we show the following two results: (1) Given a graph, the maximal vertex-induced subgraph satisfying a hereditary graph property $\pi$ can be found in time $O\left(\Delta^{\lambda(\pi)} T_{\pi}(n)(\log n)^{2}\right)$ using a polynomial number of processors, where $\lambda(\pi)$ is the maximum of diameters of minimal graphs violating $\pi$ and $T_{\pi}(n)$ is the time needed to decide whether a graph with $n$ vertices satisfies $\pi$. (2) Given a family $C=\left\{c_{1}, \ldots, c_{m}\right\}$ of subsets of a finite set $S=\{1, \ldots, n\}$ with $S=\bigcup_{i=1}^{m} c_{i}$, a minimal set cover for $S$ can be computed on an EREW PRAM in time $O\left(\alpha \beta(\log (n+m))^{2}\right)$ using a polynomial number of processors, where $\alpha=\max \left\{\left|c_{i}\right| \mid i=1, \ldots, m\right\}$ and $\beta=\max \left\{\left|d_{j}\right| \mid j=1, \ldots, n\right\}$.
\end{abstract}

\section{Introduction}

We show a way of employing the parallel algorithms for the maximal independent set problem (MIS) $[3,4,5]$ to solve problems in which maximal or minimal solutions are searched. For the bounded degree maximal subgraph problems, we have constructed an NC algorithm by employing the NC algorthms for MIS [8]. This paper extends the technique developed in [8] and gives $\mathrm{NC}$ algorithms for two kinds of such problems.

The first problem is to find a maximal set of vertices which induces a subgraph satisfying a given graph property $\pi$. The other is the minimal set cover problem that is, given a collection $C=\left\{c_{1}, \ldots, c_{m}\right\}$ with $c_{i} \subset S=\{1, \ldots, n\}$, to find a collection $C^{\prime} \subseteq C$ such that every element 
in $S$ is contained in some $c \in C^{\prime}$ but no proper subcollection $C^{\prime \prime} \subset C^{\prime}$ does not have this property.

These problems are easily solved in polynomial time by straightforward greedy sequential algorithms. However, these algorithms are hardly parallelizable since they are P-complete [7]: It is shown in [6] that the lexicographically first maximal subgraph problem for a given property $\pi$ is P-complete if $\pi$ is hereditary, nontrivial and polynomial-time testable. The same fact also holds for the greedy minimal set cover algorithm.

For the maximal subgraph problem, we need some restrictions on the property to solve the problem in NC. A graph property $\pi$ is called local if the diameter of any minimal graph violating $\pi$ is bounded by some constant. For such local property $\pi$, we consider the problem of finding a maximal vertex-induced subgraph which satisfies $\pi$ and, simultaneously, whose maximum vertex degree is at most $\Delta$, where $\Delta$ is a given constant. We prove that this problem can be solved in NC by using MIS if $\pi$ is testable in NC.

For the minimal set cover problem, we also show an algorithm which employs an MIS algorithm. This algorithm can be implemented on an EREW PRAM in time $O(\alpha \beta(\log (n+$ $m))^{2}$ ) using a polynomial number of processors, where $\alpha=\max \left\{\left|c_{i}\right| \mid i=1, \ldots, m\right\}$ and $\beta=\max \left\{\left|d_{j}\right| \mid j=1, \ldots, n\right\}$ with $d_{j}=\left\{c_{i} \mid j \in c_{i}\right\}$. This implies that if $\alpha \beta=O\left((\log (n+m))^{k}\right)$ then the problem is solvable in NC.

The algorithms for these problems are described by a scheme which applies MIS repeatedly. Thus we do not directly deal with parallelization of the problems. Our concern is how to employ an MIS algorithm to solve problems in parallel.

\section{Maximal subgraph problem for a local property}

Let $G=(V, E)$ be a graph. For a subset $U$ of vertices, the induced subgraph of $U$ is the graph defined by $G[U]=(U, E[U])$, where $E[U]$ consists of edges whose endpoints are both in $U$.

Let $\pi$ be a property on graphs. We say that a graph $G=(V, E)$ is a minimal graph violating $\pi$ with respect to vertices if $G$ violates $\pi$ and the vertex-induced subgraph $G[U]$ of $U$ satisfies $\pi$ for every subset $U$ of $V$ with $U \neq V$. The property $\pi$ is called local with respect to vertices if $\lambda(\pi)=\sup \{\operatorname{diameter}(G) \mid G$ is a minimal graph violating $\pi$ with respect to vertices $\}$ is finite.

Remark 1 A minimal graph violating a property $\pi$ with respect to vertices must be connected if $\pi$ is local.

A property $\pi$ on graphs is called hereditary with respect to vertices if for every graph $G=(V, E)$ satisfying $\pi$, the vertex-induced subgraph $G[U]$ also satisfies $\pi$ for every subset 
$U \subseteq V$

Theorem 1 Let $\pi$ be a graph property which is local and hereditary with respect to vertices. Then a maximal subgraph of a graph $G=(V, E)$ which satisfies $\pi$ and whose maximum degree is at most $\Delta$ can be computed on an EREW PRAM in time $O\left(\Delta^{\lambda(\pi)} T_{\pi}(n)(\log n)^{2}\right)$ using a polynomial number of processors, where $T_{\pi}(n)$ is the time needed to decide whether a graph with $n$ vertices satisfies $\pi$.

Proof. For subsets $W$ and $U$ of vertices with $W \cap U=\emptyset$, let $E_{U}^{W}=\{\{v, w\} \subseteq W \mid$ $\operatorname{dist}_{G[U \cup\{v, w\}]}(v, w) \leq \lambda(\pi)$ with $\left.v \neq w\right\}$ and $N_{U}(w)=\left\{u \in U \mid \operatorname{dist}_{G[U]}(u, w) \leq \lambda(\pi)-1\right\}$, where $\operatorname{dist}_{G}(\{v, w\})$ is the length of the shortest path between $v$ and $w$ in $G$. Then let $H_{U}^{W}=\left(W, E[W] \cup E_{U}^{W}\right)$. The required set $U$ of vertices is computed together with a set $W$ of vertices such that $W \cap U=\emptyset$. Initially let $W=V$ and $U=\emptyset$. At each iteration of the algorithm, a maximal independent set $I$ of $H_{U}^{W}$ is computed and added to $U$ while vertices which induce a graph violating $\pi$ or make the degree of some vertex greater than $\Delta$ are deleted from $W$ together with $I$. This is iterated $\Delta^{\lambda(\pi)}$ times. Formally the algorithm is described as follows:

1 begin $/ * G=(V, E)$ is an input $* /$

$2 \quad W \leftarrow V ; U \leftarrow \emptyset$

3 while $W \neq \emptyset$ do

4 begin

$5 \quad$ Find a maximal independent set $I$ of $H_{U}^{W}$;

$6 \quad U \leftarrow U \cup I$

$7 \quad W \leftarrow W-I$;

$8 \quad W \leftarrow W-\{w \in W \mid G[U \cup\{w\}]$ violates $\pi$ or $\operatorname{deg}(G[U \cup\{w\}])>\Delta\}$

$9 \quad$ end

10 end

$E_{U}^{W}$ consists of unordered pairs of vertices such that if the vertices are added to $U$ at the same iteration the induced subgraph of $U$ may violate the property. We show that this algorithm computes a maximal subset $U$ whose induced subgraph satisfies $\pi$ and maximum degree is at most $\Delta$.

Let $W_{0}=V$ and $U_{0}=\emptyset$. Then the graph $H_{U_{0}}^{W_{0}}$ is the same as $G=(V, E)$. Therefore in the first iteration, a maximal independent set of $G$ is computed at line 5 . For $i=1, \ldots, \Delta^{\lambda(\pi)}$, let $U_{i}, I_{i}$ and $W_{i}$ be the contents of variables $U, I$ and $W$ at the end of $i$ th iteration, respectively. 
Obviously, $W_{i} \cap U_{i}=\emptyset$ for $i=0, \ldots, \Delta^{\lambda(\pi)}$. We assume that the induced subgraph $G\left[U_{i-1}\right]$ satisfies $\pi$ and the maximum degree of $G\left[U_{i-1}\right]$ is at most $\Delta$.

Let $\{w, u\}$ be an edge in $E$ with $w \in W_{i}$ and $u \in U_{i}$. Line 8 deletes every vertex which is adjacent to more than $\Delta$ vertices in $U_{i}$ or adjacent to a vertex $v$ in $U_{i}$ with $\operatorname{deg}_{G\left[U_{i}\right]}(v)=\Delta$. Therefore $u$ is adjacent to at most $\Delta$ vertices in $U_{i}$ and $\operatorname{deg}_{G\left[U_{i} \cup\{w\}\right]}(u) \leq \Delta$. Moreover, $\left|N_{U_{i}}(w)\right| \leq \Delta^{\lambda(\pi)-1}$. Hence, for each $w$ in $W_{i}$, we see that

$$
A_{i}(w)=\sum_{u \in N_{U_{i}}(w)} \operatorname{deg}_{G\left[U_{i} \cup\{w\}\right]}(u) \leq \Delta^{\lambda(\pi)} .
$$

To show that $W$ becomes empty within $\Delta^{\lambda(\pi)}$ iterations of the while-loop, it suffices to prove that

$$
A_{i}(w)>A_{i-1}(w)
$$

for each $w$ in $W_{i}$. Since $w$ is not in the maximal independent set $I_{i}$ of $H_{U_{i-1}}^{W_{i-1}}$ computed by line $5, w$ is adjacent to a vertex $v$ in $I_{i} \subseteq W_{i-1}$ via an edge $\{w, v\}$ in $E\left[W_{i-1}\right]$ or $E_{U_{i-1}}^{W_{i-1}}$.

Case 1. $\{w, v\} \in E\left[W_{i-1}\right]$ : Then $\{w, v\}$ is an edge in $G\left[U_{i} \cup\{w\}\right]$. Hence $\operatorname{deg}_{G\left[U_{i} \cup\{w\}\right]}(v) \geq$ 1. Since $v \in N_{U_{i}}(w)$ and $v \notin N_{U_{i-1}}(w)$, we see that $A_{i}(w) \geq A_{i-1}(w)+\operatorname{deg}_{G\left[U_{i} \cup\{w\}\right]}(v)>$ $A_{i-1}(w)$.

Case 2. $\{w, v\} \in E_{U_{i-1}}^{W_{i-1}}$ : Then there is a path $w, u_{1}, \ldots, u_{k-1}, v$ with $k \leq \lambda(\pi)$ and $u_{j} \in U_{i-1}(j=1, \ldots, k-1)$ in $G\left[U_{i-1} \cup\{w, v\}\right]$. Since $v \in W_{i-1}, W_{i-1} \cap U_{i-1}=\emptyset$ and $w \neq v$, we see $v \notin U_{i-1} \cup\{w\}$. Hence $\left\{v, u_{k-1}\right\}$ is not an edge in $G\left[U_{i-1} \cup\{w\}\right]$. On the other hand, $v$ is in $U_{i}$ and $u_{k-1}$ is in $U_{i-1} \subseteq U_{i}$. Hence $\left\{v, u_{k-1}\right\}$ is an edge in $G\left[U_{i} \cup\{w\}\right]$. Therefore $\operatorname{deg}_{G\left[U_{i} \cup\{w\}\right]}\left(u_{k-1}\right)>\operatorname{deg}_{G\left[U_{i-1} \cup\{w\}\right]}\left(u_{k-1}\right)$. Since $u_{k-1} \in N_{U_{i-1}}(w) \subset N_{U_{i}}(w)$, we see that $A_{i}(w)>A_{i-1}(w)$.

We now show that $\operatorname{deg}\left(G\left[U_{i}\right]\right) \leq \Delta$ and $G\left[U_{i}\right]$ satisfies $\pi$.

Claim 1. $\operatorname{deg}\left(G\left[U_{i}\right]\right) \leq \Delta$.

Proof. For a vertex $u$ in $U_{i-1}$, if $u$ is adjacent to a vertex $w$ in $I_{i}$ via an edge in $E$, then no other vertex in $I_{i}$ is adjacent to $u$ since $I_{i}$ is also an independent set with respect to $E_{U_{i-1}}^{W_{i-1}}$. Therefore the degree of $u$ in $G\left[U_{i-1} \cup I_{i}\right]$ remains to be at most $\Delta$ since $\operatorname{deg}\left(G\left[U_{i-1} \cup\{w\}\right]\right) \leq \Delta$ by the algorithm. For a vertex $u$ in $I_{i}, \operatorname{deg}_{G\left[U_{i-1} \cup I_{i}\right]}(u)$ is at most $\Delta$ since $u$ is adjacent to at most $k$ vertices in $U_{i-1}$ and since $I_{i}$ is an independent set with respect to $E\left[W_{i-1}\right]$. Hence $\operatorname{deg}_{G\left[U_{i-1} \cup I_{i}\right]}(u) \leq \Delta$.

Claim 2. $G\left[U_{i}\right]$ satisfies $\pi$.

Proof. We assume that $G\left[U_{i}\right]$ does not satisfy $\pi$. Then, there is a minimal subset $S \subseteq U_{i}$ such that $G[S]$ violates $\pi$. Since $S \subseteq U_{i}$ and $U_{i}=U_{i-1} \cup I_{i}$, we see that $S=\left(S \cap U_{i-1}\right) \cup\left(S \cap I_{i}\right)$. The set $S \cap I_{i}$ contains at least two vertices since if $S \cap I_{i}$ consists of only one vertex then 
line 8 deletes the vertex at the last iteration. Therefore there are two distinct vertices $v, w$ such that $\{v, w\} \in E$ or there are at most $\lambda(\pi)-1$ vertices in $S \cap U_{i-1}$ which construct a path between $v$ and $w$ since $\operatorname{diameter}(G[S]) \leq \lambda(\pi)$. For each case, $\{v, w\}$ are in $E\left[W_{i-1}\right]$ or $E_{U_{i-1}}^{W_{i-1}}$ since $v, w \in I_{i} \subset W_{i-1}$. It contradicts the fact that $v, w \in S \cap I_{i} \subset I_{i}$ and $I_{i}$ is a maximal independent set with respect to $E\left[W_{i}\right] \cup E_{U_{i-1}}^{W_{i-1}}$. Hence $G\left[U_{i}\right]$ satisfies $\pi$.

Since only vertices which violate the property $\pi$ or the condition of maximum degree $\Delta$ are deleted from $W$ and since $\pi$ is hereditary, the resulting set $U$ is a maximal subset which induces a subgraph satisfying $\pi$ when $W$ becomes empty.

MIS can be solved on an EREW PRAM in $O\left((\log n)^{2}\right)$ time using a polynomial number of processors [5]. It is not hard to see that the steps other than MIS can also be implemented on an EREW PRAM in $O\left((\log n)^{2}\right)$ time using a polynomial number of processors. Hence the total algorithm can be implemented using the same amount of time and processors.

Remark 2 At line 8 of the algorithm, for each $w \in W$, it is sufficient to decide whether $G\left[N_{U}(w) \cup\{w\}\right]$ satisfies $\pi$ and $\operatorname{deg}\left(G\left[N_{U}(w) \cup\{w\}\right]\right) \leq \Delta$. Therefore, the time needed to compute line 8 depends only on $\Delta$ and $\lambda(\pi)$.

Finding a maximal subgraph of maximum degree $k$ takes $O\left(k^{2}(\log n)^{2}\right)$ time using a polynomial number of processors [8]. This is a special case of Theorem 1 for $\pi=$ "maximum degree $k ", \lambda(\pi)=2$ and $\Delta=k$. For a graph of maximum degree $\Delta$ and $\pi=$ " $k$ cycle free", it takes $O\left(\Delta^{\lfloor k / 2\rfloor}(\log n)^{2}\right)$ time to find a maximal subgraph satisfying $\pi$ of maximum degree $\Delta$ since $\lambda(\pi)=\lfloor k / 2\rfloor$.

\section{Solving the minimal set cover problem using MIS}

Let $C=\left\{c_{1}, \ldots, c_{m}\right\}$ be a family of subsets of a finite set $S=\{1, \ldots, n\}$. A subset $S^{\prime}$ of $S$ is called a hitting set for $C$ if $c_{i} \cap S^{\prime} \neq \emptyset$ for all $i=1, \ldots, m$. A subset $S^{\prime \prime}$ of $S$ is called a co-hitting set if $c_{i} \nsubseteq S^{\prime}$ for all $i=1, \ldots, m$. We say that $C$ is a set cover if $\bigcup_{i=1}^{n} c_{i}=S$.

It should be noticed that $S^{\prime}$ is a hitting set for $C$ and only if $S-S^{\prime}$ is a co-hitting set for $C$. Therefore, $S^{\prime}$ is a minimal hitting set for $C$ if and only if $S-S^{\prime}$ is a maximal co-hitting set for $C$.

The problem of finding a hitting set is closely related to the set cover problem. For a family $C=\left\{c_{1}, \ldots, c_{m}\right\}$ with $\bigcup_{i=1}^{n} c_{i}=\{1, \ldots, n\}$, let

$$
d_{j}=\left\{c_{i} \mid j \in c_{i} \in C\right\}
$$


for $j=1, \ldots, n$. Then each $d_{j}$ is not empty. Let $D=\left\{d_{1}, \ldots, d_{n}\right\}$ and $C^{\prime} \subseteq C$ be a minimal hitting set for $D$. Then $d_{j} \cap C^{\prime} \neq \emptyset$ for each $j=1, \ldots, n$. Therefore there is some $c_{i} \in d_{j} \cap C^{\prime}$. Thus $j \in c_{i}$. Hence $C^{\prime}$ is a set cover of $\{1, \ldots, n\}$ and also can be seen that $C^{\prime}$ is minimal.

Theorem 2 Let $C=\left\{c_{1}, \ldots, c_{m}\right\}$ be a family of distinct subsets of a finite set $S=\{1, \ldots, n\}$. Let $\alpha=\max \left\{\left|c_{i}\right| \mid i=1, \ldots, m\right\}$ and $\beta=\max \left\{\left|d_{j}\right| \mid j=1, \ldots, n\right\}$, where $d_{j}=\left\{c_{i} \mid j \in\right.$ $\left.c_{i}\right\}$. Then a minimal hitting set for $C$ can be computed on an EREW PRAM in time $O\left(\alpha \beta(\log (n+m))^{2}\right)$ using a polynomial number of processors with respect to $n$ and $m$.

Hence, if $\alpha \beta=O\left((\log (n+m))^{k}\right)$, then a minimal hitting set can be computed in NC.

Proof. We consider the following algorithm that finds a maximal co-hitting set for $C_{0}$ :

/* A family $C_{0}=\left\{c_{1}, \ldots, c_{m}\right\}$ with $c_{i} \subseteq S_{0}=\{1, \ldots, n\}$ for $i=1, \ldots, m$ is given. $* /$

/* We assume that $S_{0}=\bigcup_{c \in C_{0}} c$ and $\left|c_{i}\right| \geq 2$ for $i=1, \ldots, m . * /$

1 begin

$2 \quad S \leftarrow S_{0} ; C \leftarrow C_{0} ;$

$3 \quad W \leftarrow \emptyset ; / * W$ gets a maximal co-hitting set $* /$

$4 \quad$ while $S \neq \emptyset$ do

5 begin

6

$$
\begin{aligned}
& E \leftarrow \emptyset ; \\
& \operatorname{par} c \in C \text { do }
\end{aligned}
$$

\section{begin}

Choose two distinct vertices $v, w$ from $c \cap S$;

Add the edge $\{v, w\}$ to $E$ end;

Find a maximal independent set $I$ of the graph $G=(S, E)$;

22 end

$$
W \leftarrow W \cup I
$$$$
S \leftarrow S-I ;
$$$$
U \leftarrow\{u \in S \mid c \cap S \subseteq W \cup\{u\} \text { for some } c \in C\} \text {; }
$$$$
\text { par } c \in C \text { do if } c \cap U \neq \emptyset \text { then delete } c \text { from } C \text {; }
$$

$S \leftarrow S-U$;

$V \leftarrow S-\bigcup_{c \in C} c$

$W \leftarrow W \cup V$

$S \leftarrow S-V ;$

end 
The variable $W$ gets a maximal co-hitting set. Let $I_{i}, C_{i}, U_{i}, W_{i}$ and $S_{i}$ be the contents of the variables $I, C, U, W$ and $S$ just after the $i$ th iteration of the while-loop, respectively. For convenience, let $W_{0}=\emptyset$ and $U_{0}=\emptyset$. Let $U_{i}^{*}=U_{0} \cup \cdots \cup U_{i}$. We also let $E_{i}$ be the set of edges constructed during lines $7-11$. Then from the algorithm we can easily see that $S_{0}$, $S_{i-1}$ and $W_{i}$ are represented as the following disjoint unions (Figure 1):

(1) $S_{i} \cup W_{i} \cup U_{i}^{*}=S_{0}$

(2) $S_{i-1}=I_{i} \cup U_{i} \cup V_{i} \cup S_{i}$.

(3) $W_{i}=W_{i-1} \cup I_{i} \cup V_{i}$.

Claim 1. For $c \in C_{i}, c \cap S_{i}$ contains at least two elements.

Proof. By the assumption on the input, Claim 1 obviously holds for $i=0$. Assume that the claim holds for $i$ and $S_{i+1} \neq \emptyset$. Let $c$ be in $C_{i}$. Then $c \cap U_{i}=\emptyset$ from line 16 and $c \cap V_{i}=\emptyset$ from line 18. Therefore from (2) we see that $c \cap S_{i}=c \cap\left(S_{i-1}-I_{i}\right)$. If $c \cap S_{i}=\emptyset$, then $U_{i}=S_{i-1}-I_{i}$ from line 15. This yields $S_{i}=\emptyset$ from line 17 . This is a contradiction since $S_{i}$ is assumed not empty. On the other hand, if $c \cap S_{i}=\{u\}$, then $c \cap S_{i} \subseteq W_{i-1} \cup I_{i} \cup\{u\}$. This means that $u$ is in $U_{i}$ and, therefore, $c \cap U_{i} \neq \emptyset$, a contradiction. Thus $\left|c \cap S_{i}\right| \geq 2$.

Claim 2. $W_{i}$ is a co-hitting set for $C_{0}$.

Proof. We assume that $S_{i-1} \neq \emptyset$. Obviously, $W_{0}=\emptyset$ is a co-hitting set for $C_{0}$. Assume that $W_{i-1}$ is a co-hitting set for $C_{0}$. Let $c$ be in $C_{0}$.

Case 1. $c \notin C_{i}$ : $c$ was deleted during the $j$ th iteration for some $1 \leq j \leq i$. Then $c \cap U_{j} \neq \emptyset$. Hence there is $u$ in $c \cap U_{j} \subseteq U_{i}^{*}$. By (1) $u$ is not in $W_{i}$. Therefore we have $c \nsubseteq W_{i}$.

Case 2. $c \in C_{i}: c$ is also in $C_{i-1}$. Then by Claim 1 there are $v, w$ in $c \cap S_{i-1}$ with $v \neq w$ and $\{v, w\} \in E_{i}$. Since $I_{i}$ is an independent set, $v \notin I_{i}$ or $w \notin I_{i}$. Since $W_{i-1}$ is a co-hitting set for $C_{0}$, we have $c \nsubseteq W_{i-1}$. Since no element in $S_{i-1}$, hence no element in $I_{i}$, is in $W_{i-1}, v$ or $w$ is not in $W_{i-1} \cup I_{i}$. Therefore $c \nsubseteq W_{i-1} \cup I_{i}$. On the other hand, $c \cap V_{i}=\emptyset$ by line 18 . Therefore $c \nsubseteq W_{i-1} \cup I_{i} \cup V_{i}=W_{i}$.

Claim 3. For any $u \in U_{i}$, there is $c \in C_{i-1}$ such that $c \subseteq W_{i}$.

Proof. By line 15, for $u \in U_{i}$ there is $c \in C_{i-1}$ such that $c \cap\left(S_{i-1}-I_{i}\right) \subseteq W_{i-1} \cup I_{i} \cup\{u\}$. Then $c \cap S_{i-1} \subseteq W_{i-1} \cup I_{i} \cup\{u\}$. Note that for $c \in C_{i-1}$ we have $c \cap U_{i-1}^{*}=\emptyset$ by line 16 . Then

$$
\begin{aligned}
c & =c \cap\left(S_{i-1} \cup W_{i-1} \cup U_{i-1}^{*}\right. & & \text { (by }(1)) \\
& =\left(c \cap S_{i-1}\right) \cup\left(c \cap W_{i-1}\right) \cup\left(c \cap U_{i-1}^{*}\right) & & \\
& \subseteq W_{i-1} \cup I_{i} \cup\{u\} . & & \text { by } \left.c \cap U_{i-1}^{*}=\emptyset\right)
\end{aligned}
$$

Let $t$ be the integer such that $S_{t}=\emptyset$. Then by (1) $S_{0}=W_{t} \cup U_{t}^{*}$. From Claim $2 W_{t}$ is a co-hitting set. Claim 3 asserts that for any $u \in U_{t}^{*}$ there is some $c$ with $c \subseteq W_{t} \cup\{u\}$. Therefore $W_{t}$ is a maximal co-hitting set for $C_{0}$.

Claim 4. $t \leq \alpha \beta$. 


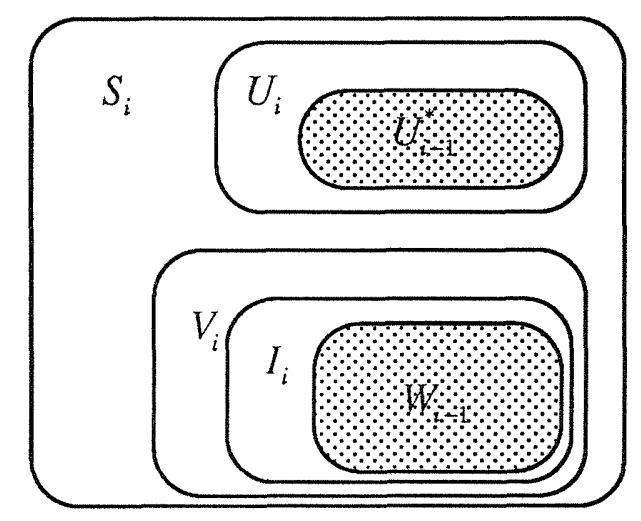

Figure 1: Relation between $S_{i}, I_{i}, V_{i}, W_{i-1}$ and $U_{i}^{*}$

Proof. For $u \in S_{i}$, we define

$$
B_{i}(u)=\left\{v \mid u \neq v \text { and }\{u, v\} \subseteq c \cap S_{i} \text { for some } c \in C_{i}\right\}
$$

It is easy to see that $\left|B_{i}(u)\right| \leq \alpha \beta$. Then it suffices to show that

$$
\left|B_{i}(u)\right|<\left|B_{i-1}(u)\right|
$$

for each $u \in S_{i}$. If $u \in S_{i}$, then $u$ is not in $I_{i}$ from line 14. Since $I_{i}$ is a maximal independent set, there is $v$ with $\{u, v\} \in E_{i}$. Therefore $\{u, v\} \subseteq c \cap S_{i-1}$ for some $c \in C_{i-1}$. Hence $v$ is in $B_{i-1}(u)$. However, $v$ is not in $S_{i}$ since $v$ is in $I_{i}$. Therefore $v$ is not in $B_{i}(u)$.

As in the proof of Theorem 1, the part of finding a maximal independent set can be implemented on an EREW PRAM in $\left.O\left((\log (n+m))^{2}\right)\right)$ time using polynomially many processors with respect to $n$ and $m$. The other steps can also be implemented with at most the same amount of time and processors.

The following corollary is obtained in a straightforward way from Theorem 2:

Corollary 1 Let $C=\left\{c_{1}, \ldots, c_{m}\right\}$ be a family of subsets of a finite set $S=\{1, \ldots, n\}$ such that $S=\bigcup_{i=1}^{m} c_{i}$. Let $\alpha=\max \left\{\left|c_{i}\right| \mid i=1, \ldots, m\right\}$ and $\beta=\max \left\{\left|d_{j}\right| \mid j=1, \ldots, n\right\}$, where $d_{j}=\left\{c_{i} \mid j \in c_{i}\right\}$. Then a minimal set cover for $S$ can be computed on an EREW PRAM in time $O\left(\alpha \beta(\log (n+m))^{2}\right)$ using a polynomial number of processors with respect to $n$ and $m$.

Hence, if $\alpha \beta=O\left((\log (n+m))^{k}\right)$, then a minimal set cover can be computed in NC.

Remark 3 An NC approximation algorithm for the set cover problem is shown in [1]. But it should be noted here that their algorithm does not produce a minimal set cover. 


\section{Conclusion}

We have shown that parallel MIS algorithms are useful to solve the minimal set cover problem and the maximal subgraph problem for a property "local and of degree at most $\Delta$ ". However, the idea of using MIS does not seem to work for other properties, for example, "acyclic", "planar", which are not local. MIS locates at an interesting position in the NC hierarchy. It is in $\mathrm{NC}^{2}$ but unlikely to belong to classes such as $\mathrm{AC}^{1}$ and DET shown in [2]. It is not difficult to see that the algorithms shown in this paper can be transformed to $\mathrm{NC}^{1}$-reductions to MIS. Hence the results in this paper give some new problems $\mathrm{NC}^{1}$-reducible to MIS.

\section{References}

[1] B. Berger, J. Rompel and P.W. Shor, Efficient NC algorithm for set cover with application to learning and geometry, Proc. 30th IEEE FOCS (1989) 54-59.

[2] S.A. Cook, Taxonomy of problems with fast parallel algorithms, Inform. Contr. 64 (1985) $2-22$.

[3] M. Goldberg and T. Spencer, A new parallel algorithm for the maximal independent set problem, SIAM J. comput. 18 (1989) 419-427.

[4] R.M. Karp and A. Wigderson, A fast parallel algorithm for the maximal independent set problem, J. Assoc. Comput. Mach. 32 (1985) 762-773.

[5] M. Luby, A simple parallel algorithm for the maximal independent set problem, Proc. 17th ACM STOC (1985) 1-10.

[6] S. Miyano, The lexicographically first maximal subgraph problems: P-completeness and NC algorithms, Math. Systems Theory 22 (1989) 47-73.

[7] S. Miyano, S. Shiraishi and T. Shoudai, A list of P-complete problems, RIFIS-TR-CS17, Research Institute of Fundamental Information Science, Kyushu University, 1989 (revised in December, 1990).

[8] T. Shoudai and S. Miyano, Bounded degree maximal subgraph problems are in NC, Proc. Toyohashi Symposium on Theoretical Computer Science (1990) 97-101. 